\title{
STATIONARY AND DYNAMICAL SOLUTIONS OF THE GROSS-PITAEVSKII EQUATION FOR A BOSE-EINSTEIN CONDENSATE IN A $\mathcal{P} \mathcal{T}$ SYMMETRIC DOUBLE WELL
}

\author{
Holger Cartarius*, Dennis Dast, Daniel Haag, Günter Wunner, \\ RÜDIGER EichleR, Jorg MAin
}

\author{
Institut für Theoretische Physik 1, Universität Stuttgart, Pfaffenwaldring 57, 70550 Stuttgart, Germany \\ * corresponding author: Holger.Cartarius@itp1.uni-stuttgart.de
}

\begin{abstract}
We investigate the Gross-Pitaevskii equation for a Bose-Einstein condensate in a $\mathcal{P} \mathcal{T}$ symmetric double-well potential by means of the time-dependent variational principle and numerically exact solutions. A one-dimensional and a fully three-dimensional setup are used. Stationary states are determined and the propagation of wave function is investigated using the time-dependent GrossPitaevskii equation. Due to the nonlinearity of the Gross-Pitaevskii equation the potential depends on the wave function and its solutions decide whether or not the Hamiltonian itself is $\mathcal{P} \mathcal{T}$ symmetric. Stationary solutions with real energy eigenvalues fulfilling exact $\mathcal{P} \mathcal{T}$ symmetry are found as well as $\mathcal{P} \mathcal{T}$ broken eigenstates with complex energies. The latter describe decaying or growing probability amplitudes and are not true stationary solutions of the time-dependent Gross-Pitaevskii equation. However, they still provide qualitative information about the time evolution of the wave functions.
\end{abstract}

Keywords: Bose-Einstein condensates, $\mathcal{P} \mathcal{T}$ symmetry, Gross-Pitaevskii equation, stationary states, dynamics.

\section{INTRODUCTION}

Bose-Einstein condensates can, at extremely low temperatures, be described by the Gross-Pitaevskii equation [1, 2], which reads in a particle number scaled form and in appropriate units

$$
\mathrm{i} \dot{\psi}(\boldsymbol{x}, t)=\left(-\Delta+V(\boldsymbol{x})-g|\psi(\boldsymbol{x}, t)|^{2}\right) \psi(\boldsymbol{x}, t) .
$$

This is the Hartree approximation of the corresponding many-particle equation for the dilute atomic gas, where the assumption that all atoms are in the quantum mechanical ground state is used. An external potential, in which the atom cloud is trapped, is described by $V(x)$. Additionally, the atoms interact via the short-range van der Waals force. In the dilute gas it can be described sufficiently exact by an s-wave scattering process, which leads in the mean-field approximation to the nonlinear contribution $-g|\psi(\boldsymbol{x}, t)|^{2}$. The strength $g$ is determined by the s-wave scattering length. Using magnetic fields acting on the hyperfine levels of the atoms and exploiting Feshbach resonances, the scattering length can be tuned and $g$ is a true parameter of the system, which can be varied.

Bose-Einstein condensates are a promising candidate for a first experimental realization of a special class of non-Hermitian Hamiltonians in quantum mechanics, viz. systems which possess a $\mathcal{P} \mathcal{T}$ symmetry in spite of their non-Hermitian nature. As was shown first by Bender and Boettcher, these Hamiltonians exhibit remarkable properties [3] such as stationary states with real eigenvalues in the presence of loss and gain terms in the potential.
With the operators of spatial reflection $\mathcal{P}: x \rightarrow-x$, $p \rightarrow-p$, and of time reversal $\mathcal{T}: x \rightarrow x, p \rightarrow-p$, $\mathrm{i} \rightarrow-\mathrm{i}$ the $\mathcal{P} \mathcal{T}$ symmetry of the Hamiltonian can be expressed in terms of the commutator relation

$$
[\mathcal{P} \mathcal{T}, H]=0
$$

Since the kinetic energy term in the Hamiltonian is always $\mathcal{P} \mathcal{T}$ symmetric one obtains in a simple calculation from (2) the necessary condition

$$
V^{*}(-x)=V(x)
$$

for the potential.

Whereas the experimental realization of $\mathcal{P} \mathcal{T}$ symmetry has successfully been achieved 4, 5, for optical wave guides 612 , a verification in a genuine quantum system is still lacking. However, a proposal for a Bose-Einstein condensate with a $\mathcal{P} \mathcal{T}$ symmetric external potential was given by Klaiman et al. [11, who suggested a double-well setup, where atoms are coherently incoupled in one well and outcoupled from the other.

In this article, we will solve the Gross-Pitaevskii equation of such a system and show that it indeed supports $\mathcal{P} \mathcal{T}$ symmetric solutions. Since the model describes a true quantum system, our investigations provide a good starting point for an experimental realization of this quantum system with $\mathcal{P} \mathcal{T}$ symmetry. However, in the case of the nonlinear Gross-Pitaevskii equation (1) one has to address one additional critical question. The wave function has an influence on the nonlinear Hamiltonian's symmetry. Along with the 
external potential the interaction term $-g|\psi(\boldsymbol{x}, t)|^{2}$ has to fulfill the condition (3). The Hamiltonian is only $\mathcal{P} \mathcal{T}$ symmetric if the square modulus of its solution $|\psi(\boldsymbol{x}, t)|^{2}$ is a symmetric function of $x$. This is always fulfilled for $\mathcal{P} \mathcal{T}$ symmetric wave functions. Thus, one may state that the nonlinear Hamiltonian of the Gross-Pitaevskii equation preserves its own symmetry in the case of exact $\mathcal{P} \mathcal{T}$ symmetry. But does this happen also with nonlinearity? Wave functions describing $\mathcal{P} \mathcal{T}$ broken eigenstates usually do not possess a symmetric square modulus and destroy also the $\mathcal{P} \mathcal{T}$ symmetry of the Hamiltonian.

Previous studies of $\mathcal{P} \mathcal{T}$ symmetric systems with nonlinearity indicate that the nonlinearity does not destroy the relevant effects known from linear $\mathcal{P} \mathcal{T}$ symmetric Hamiltonians. An investigation of a BoseEinstein condensate in a two-mode approximation using a non-Hermitian Bose-Hubbard model was made by Graefe et al. [13 15]. Furthermore, the combination of $\mathcal{P} \mathcal{T}$ symmetry and nonlinearity has been studied in quantum mechanical model potentials [10, 16], in optics [17] or for a Bose-Einstein condensate with an idealized double- $\delta$ trap [18, a system of which the linear variant already attracted much interest [19-23].

To investigate Bose-Einstein condensates in a $\mathcal{P} \mathcal{T}$ symmetric double well we first introduce our numerical approach in Section 2. Then we present and discuss the numerical results for the energy eigenvalues, the stationary wave functions and the time evolution of non-stationary initial wave packets in Section 3 . Conclusions are drawn in Section 4

\section{NUMERICAL APPROACH TO BOSE-EINSTEIN CONDENSATES IN A $\mathcal{P} \mathcal{T}$ SYMMETRIC DOUBLE WELL}

\subsection{Gross-Pitaevski EQuation}

We consider a Bose-Einstein condensate of particles with mass $m$ in a double-well setup described by the external potential

$$
\begin{aligned}
V(\boldsymbol{x})=\frac{m}{2} \omega_{x}^{2} x^{2}+\frac{m}{2} \omega_{y, z}^{2}\left(y^{2}+z^{2}\right) & +v_{0} e^{-\sigma x^{2}} \\
& +\mathrm{i} \Gamma x \mathrm{e}^{-\rho x^{2}}
\end{aligned}
$$

where a three-dimensional harmonic trap is superimposed by a Gaussian barrier in the $x$ direction (cf. Figure 11. The trapping frequencies are $\omega_{x}$ for the direction of the double-well structure and $\omega_{y, z}$ for the two remaining directions. The barrier has its maximum at $x=0$, the height $v_{0}$, and its width is determined by $\sigma$. An outcoupling (incoupling) of atoms is reflected by a negative (positive) imaginary potential contribution in the left (right) well. Its strength is determined by the gain-loss parameter $\Gamma$. Since it affects the probability amplitude of the whole condensate, the physical interpretation is a coherent out-/incoupling. With this ansatz we are not considering individual atoms but the macroscopic wave function of the condensed phase. The potential (4)

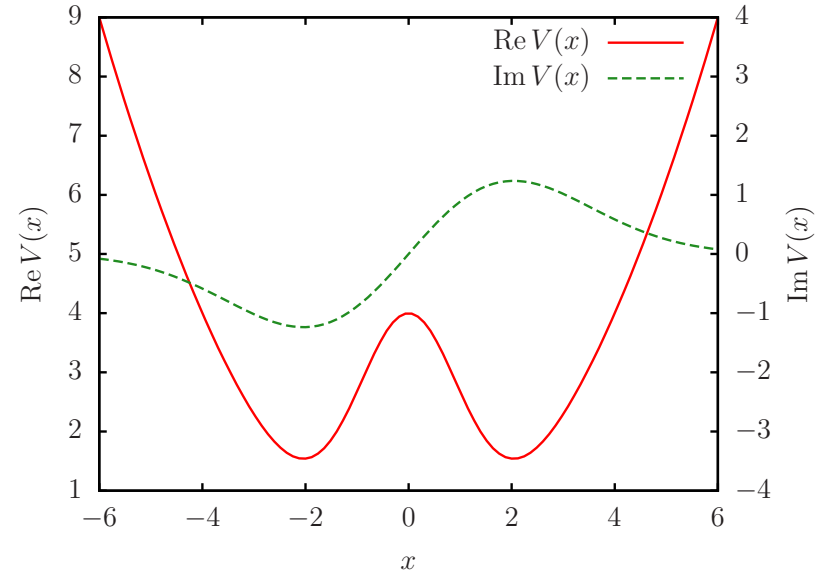

FiguRE 1. $\mathcal{P} \mathcal{T}$ symmetric external potential. The real part (solid line) defines the confinement of the condensed atom cloud and the imaginary part (dashed line) describes the gain-loss contributions due to the coherent in- and outcoupling of atoms.

has been chosen such that it fulfills the condition (3), i.e. its real part is a symmetric function of $x$, while its imaginary part is antisymmetric. Thus, the linear external potential (4) is $\mathcal{P} \mathcal{T}$ symmetric.

Introducing the length scale $a_{0}=\sqrt{\hbar / m \omega_{y, z}}$ defined by the trap frequency in the direction perpendicular to the double-well shape and the unit of energy $E_{0}=\hbar^{2} / 2 m a_{0}^{2}$ the dimensionless potential reads

$$
V(\boldsymbol{x})=\omega_{x}^{2} x^{2}+y^{2}+z^{2}+v_{0} e^{-\sigma x^{2}}+\mathrm{i} \Gamma x \mathrm{e}^{-\rho x^{2}},
$$

and the evolution of the condensate is described by the Gross-Pitaevskii equation (1). With the chemical potential $\mu$ and the usual separation ansatz $\psi(\boldsymbol{x}, t)=$ $\phi(\boldsymbol{x}) \mathrm{e}^{-\mathrm{i} \mu t}$ in the units defined above one obtains the time-independent Gross-Pitaevskii equation

$$
\left(-\Delta+V(\boldsymbol{x})-g|\phi(\boldsymbol{x})|^{2}\right) \phi(\boldsymbol{x})=\mu \phi(\boldsymbol{x}) .
$$

In our calculations we use the potential parameters $\omega_{x}=0.5, v_{0}=4$, and $\sigma=0.5$. The width parameter

$$
\rho=\frac{\sigma}{2 \ln \left(v_{0} \sigma / \omega_{x}^{2}\right)}
$$

of the gain-loss potential is chosen such that the extrema of the real and imaginary potential parts coincide, cf. Figure 1 If only the $x$ direction is considered and all $y$ and $z$ terms are removed from the potential and the wave function, we can reduce the model to one dimension that contains the relevant $\mathcal{P} \mathcal{T}$ symmetric information.

\subsection{TWO MEthods: VARIATIONAL GAUSSiAN AND NUMERICALLY EXACT}

We use two methods to solve the time-dependent and time-independent Gross-Pitaevskii equations (1) and (6). The Gaussian variational method has been shown to provide highly precise solutions with low numerical effort [24-26]. Since in our work it is applied 
for the first time to Bose-Einstein condensates in a $\mathcal{P} \mathcal{T}$ symmetric complex potential, we compare it to numerically exact solutions of the Gross-Pitaevskii equation in the one-dimensional case.

The idea of the Gaussian variational method consists of the restriction to a Gaussian shaped wave function

$$
\begin{array}{r}
\psi(\boldsymbol{z}, \boldsymbol{x})=\sum_{k=1}^{2} \mathrm{e}^{-\left[A_{x}^{k}\left(x-q_{x}^{k}\right)^{2}+A_{y, z}^{k}\left(y^{2}+z^{2}\right)\right]} \\
\times \mathrm{e}^{\mathrm{i} p_{x}^{k}\left(x-q_{x}^{k}\right)-\varphi^{k}}
\end{array}
$$

described by a small set of variational parameters, viz.

$$
\boldsymbol{z}(t)=\left\{A_{x}^{k}(t), A_{y, z}^{k}(t), q_{x}^{k}(t), p_{x}^{k}(t), \varphi^{k}\right\} .
$$

In the case of the $\mathcal{P} \mathcal{T}$ symmetric double-well setup it is reasonable to start with two Gaussian wave functions, where each of them is located in one of the wells. The widths of the Gaussians are determined by the complex parameters $A_{x}^{1}, A_{x}^{2}, A_{y, z}^{1}$ and $A_{y, z}^{2}$. Since the relevant dynamics only affects the $x$ coordinate and the trap was assumed to be symmetric in $y$ and $z$ directions, we include in our ansatz the same symmetry for the Gaussian wave functions. The positions and the corresponding momenta of both Gaussians are determined by the real coordinates $q_{x}^{1}, q_{x}^{2}, p_{x}^{1}$ and $p_{x}^{2}$. The amplitudes and phases are introduced via the complex variables $\varphi^{1}$ and $\varphi^{2}$. This leads in total to 16 real parameters completely defining the condensate wave function. Reducing the model to one dimension we end up with twelve real variables.

Certainly, the ansatz (8) cannot solve the timedependent Gross-Pitaevskii equation (1) exactly. One way to find the "best" approximative solution with a wave function restricted to the Gaussian form (8) is the application of the McLachlan time-dependent variational principle [27,

$$
\delta I=\delta\|i \chi(\boldsymbol{z}(t), \boldsymbol{x})-H \psi(\boldsymbol{z}(t), \boldsymbol{x})\|^{2} \stackrel{!}{=} 0 .
$$

In this procedure the variation with respect to the parameters in the wave function $\chi$ is performed such that the functional $I$ is minimized. In a second step one sets $\dot{\psi} \equiv \chi$ and obtains the equations of motion, which in our case are of the form

$$
\begin{aligned}
& \dot{A}_{x}^{k}=-4 \mathrm{i}\left(\left(A_{x}^{k}\right)^{2}+\left(A_{y, z}^{k}\right)^{2}\right)+\mathrm{i} V_{2 ; x}^{k}, \\
& \dot{A}_{y, z}^{k}=-4 \mathrm{i}\left(\left(A_{x}^{k}\right)^{2}+\left(A_{y, z}^{k}\right)^{2}\right)+\mathrm{i} V_{2 ; y, z}^{k}, \\
& \dot{q}_{x}^{k}= 2 p_{x}^{k}+s_{x}^{k}, \\
& \dot{p}_{x}^{k}=-\operatorname{Re} v_{1 ; x}^{k}-2 \operatorname{Im} A_{x}^{k} s_{x}^{k}-2 \operatorname{Re} V_{2 ; x}^{k} q_{x}^{k}, \\
& \dot{\varphi}^{k}=\mathrm{i} v_{0}^{k}+2 \mathrm{i}\left(A_{x}^{k}+A_{y, z}^{k}\right)-\mathrm{i}\left(p_{x}^{k}\right)^{2} \\
& \quad-\mathrm{i} p_{x}^{k} s_{x}^{k}+\mathrm{i} q_{x}^{k} v_{1 ; x}^{k}+\mathrm{i} q_{x}^{k} V_{2 ; x}^{k} q_{x}^{k},
\end{aligned}
$$

with

$$
s_{x}^{k}=\frac{1}{2}\left(\operatorname{Re} A_{x}^{k}\right)^{-1}\left(\operatorname{Im} v_{1 ; x}^{k}+2 \operatorname{Im} V_{2 ; x}^{k} q_{x}^{k}\right) .
$$

The equations of motion $11 \mathrm{a}-(11 \mathrm{f})$ contain effective potential terms $\boldsymbol{v}=\left(v_{0}^{1}, \ldots, v_{1 ; x}^{1}, \ldots, V_{2 ; x}^{1}, \ldots\right)$, which are obtained from a system of linear equations, $\boldsymbol{K} \boldsymbol{v}=\boldsymbol{r}$, where the matrix $\boldsymbol{K}$ contains weighted overlap integrals of the Gaussians and the vector $\boldsymbol{r}$ consists of weighted Gaussian averages of all potential terms including the nonlinearity. The detailed form can be found in [28]. The dynamics of the condensate wave function is found by solving the ordinary differential equations 11a)-(11f) with a Runge-Kutta algorithm [29].

Stationary states or solutions of the time-independent Gross-Pitaevskii equation are found by the requirements $\dot{A}_{x}^{k}=\dot{A}_{y, z}^{k}=\dot{q}_{x}^{k}=\dot{p}_{x}^{k}=0$ (12 conditions for real numbers $), \dot{\varphi}^{1}=\dot{\varphi}^{2}$ (2 conditions). These states have to be normalized, $\|\psi\|=1$, which is important in the nonlinear Gross-Pitaevskii equation and adds an additional constraint. Due to the arbitrary global phase one of the 16 Gaussian parameters introduced above is free and 15 parameters must be varied to fulfill the 15 conditions. This is done with a 15-dimensional root search by applying a Powell hybrid method [29]. If we consider a one-dimensional condensate the root search reduces to 11 conditions, which have to be fulfilled by 11 appropriately chosen parameters. This small difference exemplifies the high scalability of the variational Gaussian method. An increase of the dimension or the flexibility of the wave function leads only to a moderate increase of the numerical effort.

The numerically exact integrations of the GrossPitaevskii equations (1) and (6) presented in this paper are carried out for the one-dimensional setup, where the computational costs are reasonable. The stationary wave functions are integrated outward from $x=0$ in positive and negative direction using a RungeKutta algorithm with initial values $\operatorname{Re} \psi(0), \psi^{\prime}(0) \in \mathbb{C}$, and $\mu \in \mathbb{C}$. They have to be chosen such that the five conditions $\psi(\infty) \rightarrow 0, \quad \psi(-\infty) \rightarrow 0$, and $\|\psi\|=1$ are satisfied. The conditions define square-integrable and normalized wave functions, i.e. the stationary states we are interested in. Note that the arbitrary global phase has been exploited by setting $\operatorname{Im} \psi(0)=0$ for the initial values of the integration. For dynamical calculations the split operator method is used, as explained in 28].

\section{NumericAl SOLUTIONS}

\subsection{ENERGY EIGENVALUES}

Figure 2 shows a typical example for the eigenvalues of the time-independent Gross-Pitaevskii equation (6). First, one observes that the linear system $(g=0)$ reveals the known features of complex Hamiltonians with $\mathcal{P} \mathcal{T}$ symmetry. Two real eigenvalue solutions are found below a value $\Gamma_{\mathrm{EP}}$, where they merge in an exceptional point. For larger values of $\Gamma$ two complex and complex conjugate solutions are found. The agreement between the Gaussian approximation (solid 

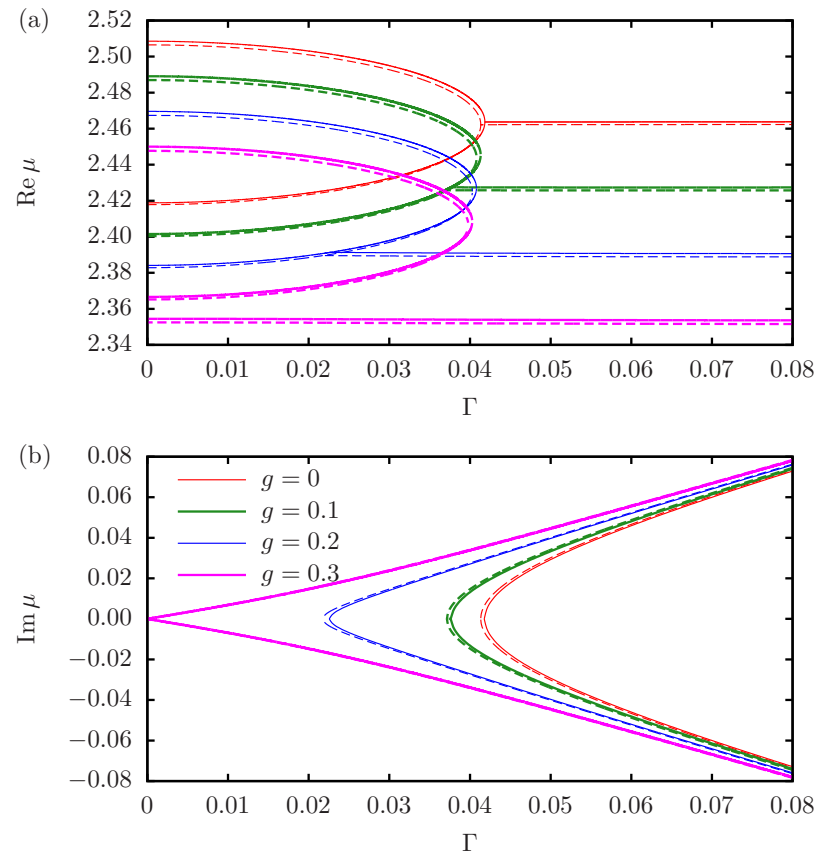

Figure 2. Eigenvalues of the time-independent GrossPitaevskii equation 6. for different values of the nonlinearity $g$ in dependence on the gain-loss parameter $\Gamma$. With increasing $g$ the real part of the energies decreases. The Gaussian approximation (solid lines) and the numerically exact solutions (dashed lines) are shown. Vanishing imaginary parts are not plotted. Two solutions with real eigenvalues are obtained up to a value $\Gamma_{\mathrm{EP}} \approx 0.04$, where they merge in an exceptional point. Two additional solutions with complex eigenvalues are obtained, starting at a critical value $\Gamma_{\mathrm{c}}$, where $\Gamma_{\mathrm{c}}<\Gamma_{\mathrm{EP}}$ for $g \neq 0$.

lines) and the numerically exact solution (dashed lines) is excellent.

An important result is the persistence of real eigenvalue solutions for nonvanishing values of the gain-loss parameter $\Gamma$ in the case $g \neq 0$. This demonstrates that the Gross-Pitaevskii equation with a nonlinearity in the potential still supports real eigenvalue solutions, i.e. non-decaying states. There are, however, a few crucial differences between the linear and the nonlinear system. The complex eigenvalue solutions are now born at a value $\Gamma_{\mathrm{c}}<\Gamma_{\mathrm{EP}}$, whereas at the exceptional point $\Gamma_{\mathrm{EP}}$ only the real eigenvalue states vanish and new complex solutions do not appear. The bifurcation scenario of the linear system seems to be split between the emergence of complex eigenvalue solutions at $\Gamma_{c}$ and the disappearance of the real eigenvalue states at $\Gamma_{\mathrm{EP}} \neq \Gamma_{\mathrm{c}}$. It is possible to explain this unusual characteristics by the non-analyticity of the GrossPitaevskii equation [28]. For sufficiently high values of $g$ the complex eigenvalues exist down to $\Gamma \rightarrow 0$ and only lose their imaginary contribution for $\Gamma=0$.

The one-dimensional model already contains the whole important potential shape for the analysis of a $\mathcal{P} \mathcal{T}$ symmetric condensate. Thus, one can expect that it includes all important features in the eigenvalue structure. This is confirmed by the comparison
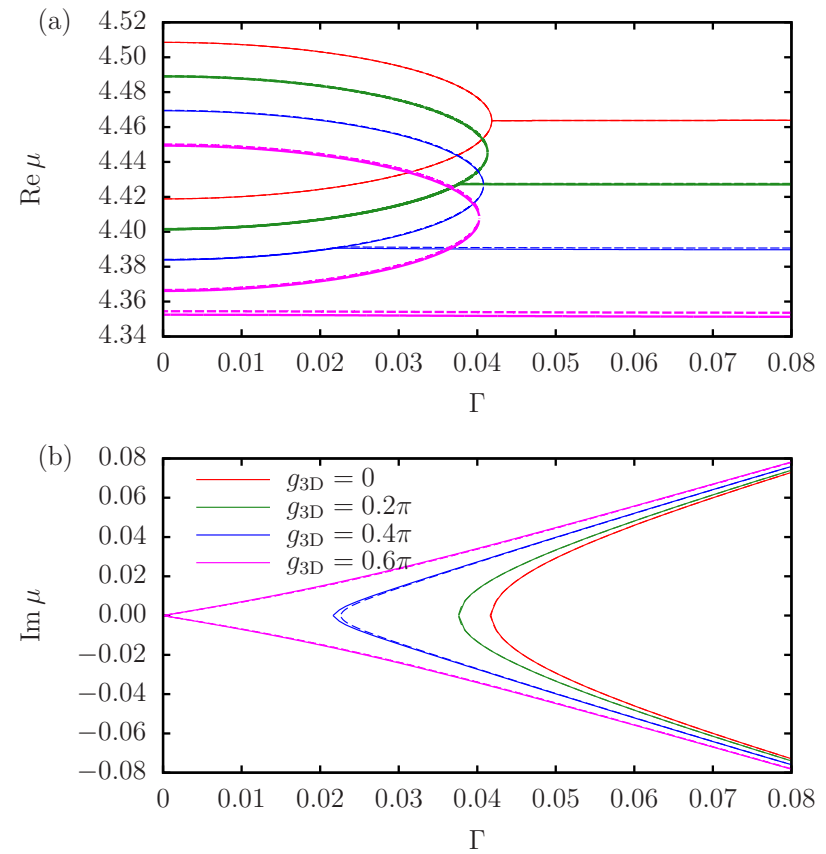

Figure 3. Real (a) and nonvanishing imaginary (b) parts of the energy eigenvalues of the time-independent Gross-Pitaevskii equation in the one- (dashed lines) and three-dimensional (solid lines) models. The comparison shows that, by applying an appropriate rescaling of the nonlinearity parameter $g$ and an energy shift for the energy contributions from the two additional directions, the one-dimensional model already provides a very good quantitative description of the fully three-dimensional setup. It is almost impossible to identify the differences in the graph.

of the eigenvalues obtained with the one- (dashed) and three-dimensional (solid) potentials in Figure 3. The three-dimensional setup contains two additional directions in which an external harmonic oscillator potential is present. This leads to the assumption that, in the ground state, the energy is shifted by a value of $\Delta \mu=2$ in the units introduced above. Furthermore, the spatial extension in $y$ and $z$ directions leads to additional energy contributions from the contact interaction. The difference can be estimated from the expectation value of the contact energy. We demand that the expectation values of both models are identical,

$$
\int_{\mathbb{R}^{3}} \mathrm{~d} x \mathrm{~d} y \mathrm{~d} z g_{3 \mathrm{D}}\left|\psi_{3 \mathrm{D}}(\boldsymbol{x})\right|^{4} \stackrel{!}{=} \int_{\mathbb{R}} \mathrm{d} x g_{1 \mathrm{D}}\left|\psi_{1 \mathrm{D}}(x)\right|^{4}
$$

and obtain the condition

$$
g_{3 \mathrm{D}}=2 \pi g_{1 \mathrm{D}}
$$

for a rescaled $g_{1 \mathrm{D}}$ of the one-dimensional model which leads to the same contact energy as a threedimensional wave function with $g_{3 \mathrm{D}}$. In this calculation we used a product ansatz $\psi_{3 \mathrm{D}}(\boldsymbol{x}) \approx$ $\psi_{1 D}(x) \psi_{0}(y) \psi_{0}(z)$ of the wave function, where $\psi_{0}$ describes the harmonic oscillator ground state [28]. This assumption is only correct in the linear case without 

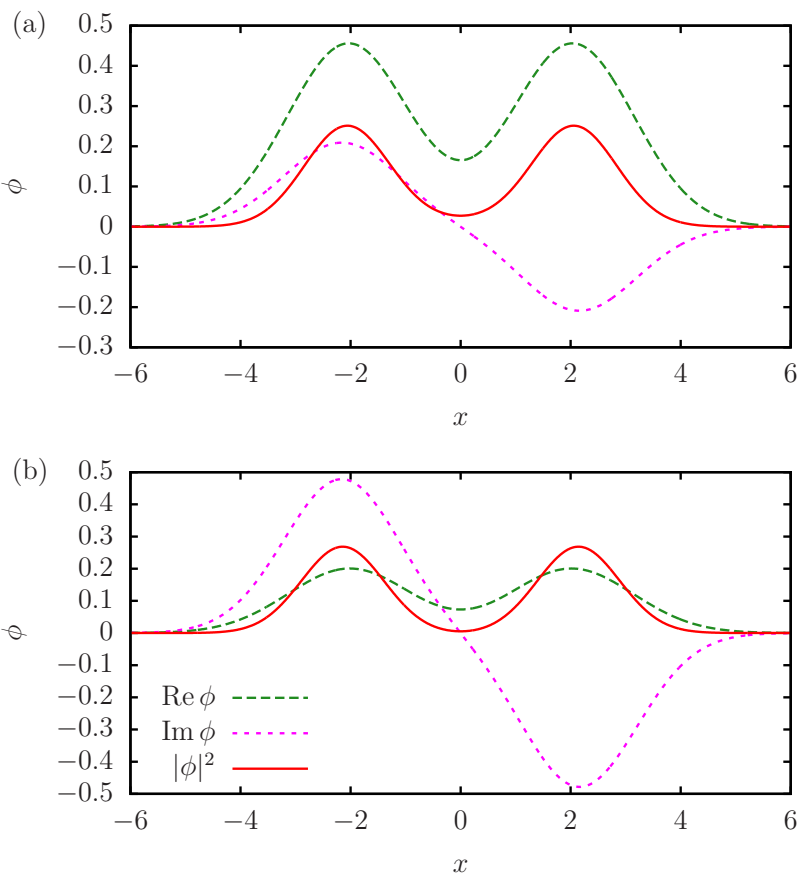

Figure 4. Wave functions of the ground (a) and excited (b) eigenstates with real eigenvalues in the case $g=0.2$ and $\Gamma=0.03$. Only the Gaussian solutions are shown since they are almost identical with the numerically exact values. The square moduli of both wave functions are symmetric functions of $x$, preserving the $\mathcal{P} \mathcal{T}$ symmetry of the nonlinear Hamiltonian.

contact interaction. The remarkable agreement of both calculations in Figure 3 shows, however, that it still approximates the nonlinear case very well even for considerable nonlinearities $g_{1 \mathrm{D}} \approx 0.3$. Due to the excellent quantitative agreement between the one- and three-dimensional calculations we will only consider the one-dimensional variant in the following sections.

\subsection{WAVE FUNCTIONS}

We have already seen that real eigenvalue solutions do appear in the nonlinear Gross-Pitaevskii equation. But, we still do not know whether we have found truly $\mathcal{P} \mathcal{T}$ symmetric solutions. As explained in the introduction, the Hamiltonian is only $\mathcal{P} \mathcal{T}$ symmetric if the square modulus of the eigenstate is a symmetric function of $x$. This question will be answered in this section.

Figure 4 shows the wave functions belonging to the real eigenvalues for $g=0.2$ and $\Gamma=0.03$. Obviously, the square modulus of both wave functions is a symmetric function of $x$. This preserves the Hamiltonian's $\mathcal{P} \mathcal{T}$ symmetry, it is not destroyed by the nonlinearity. Or in other words, the nonlinear Hamiltonian picks as eigenstates wave functions which render itself $\mathcal{P} \mathcal{T}$ symmetric.

The situation is, however, completely different for the wave functions of the states with complex eigenvalues. In linear $\mathcal{P} \mathcal{T}$ symmetric models the complex eigenvalue solutions belong to the $\mathcal{P} \mathcal{T}$ broken case, in which the wave functions do not reflect the $\mathcal{P} \mathcal{T}$
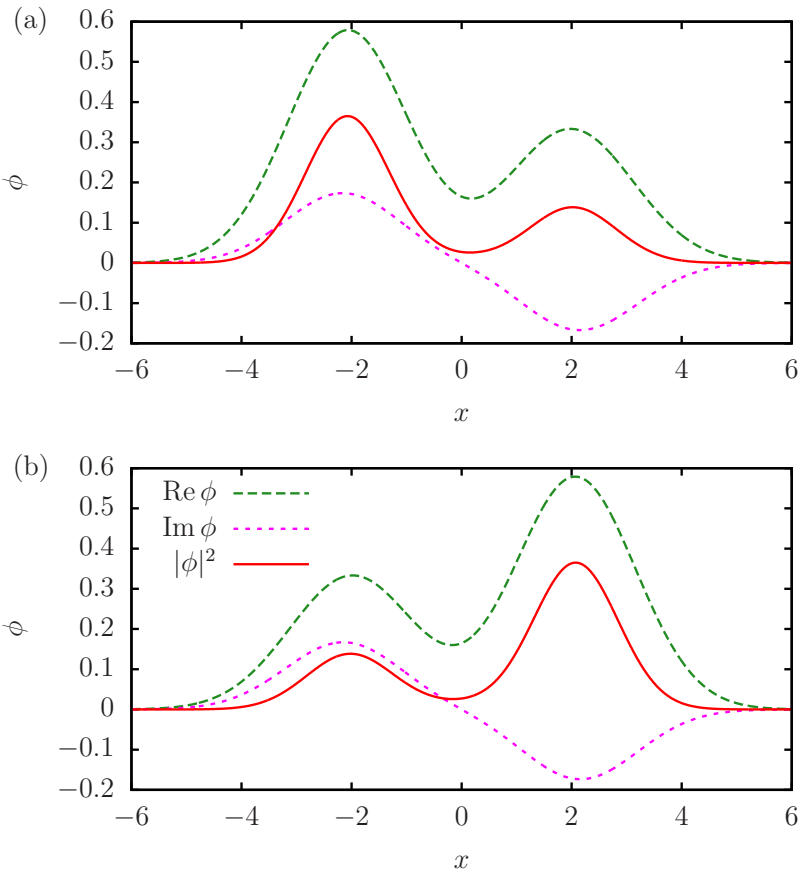

FiguRE 5. Wave functions of the complex eigenvalue solutions with negative (a) and positive (b) imaginary part in the case $g=0.2$ and $\Gamma=0.03$. Again only the Gaussian wave functions are shown. The square moduli are not symmetric functions of $x$ and destroy the Hamiltonian's $\mathcal{P} \mathcal{T}$ symmetry.

symmetry of the Hamiltonian. This is also the case for the solutions of the Gross-Pitaevskii equation as can be seen in Figure 5. The wave functions of the solutions with complex eigenvalues are not $\mathcal{P} \mathcal{T}$ symmetric. Their square moduli are not symmetric functions of $x$, and thus even the $\mathcal{P} \mathcal{T}$ symmetry of the Hamiltonian is destroyed, a circumstance which is not possible in a linear quantum system. The wave function belonging to the eigenvalue with negative imaginary part has a higher amplitude in the left well with loss, whereas the probability amplitude of the state with positive imaginary part of the energy is shifted more to the right well with gain.

A $\mathcal{P} \mathcal{T}$ symmetric Bose-Einstein condensate will only be observable if the eigenstates are stable with respect to quantum fluctuations. We performed a linear stability analysis to check the behavior of the eigenstates with real eigenvalues. A linearization of the equations of motion 11a-11f of the Gaussian parameters around the stationary states and the solution of the Bogoliubov-de Gennes equations for the numerically exact wave functions has been done [28]. We found that the excited state is stable for all combinations of $g$ and $\Gamma$ as long as it exists. The ground state, however, becomes unstable as soon as the $\mathcal{P} \mathcal{T}$ broken branches with complex eigenvalues emerge in the energy diagram of Figure 2 . 

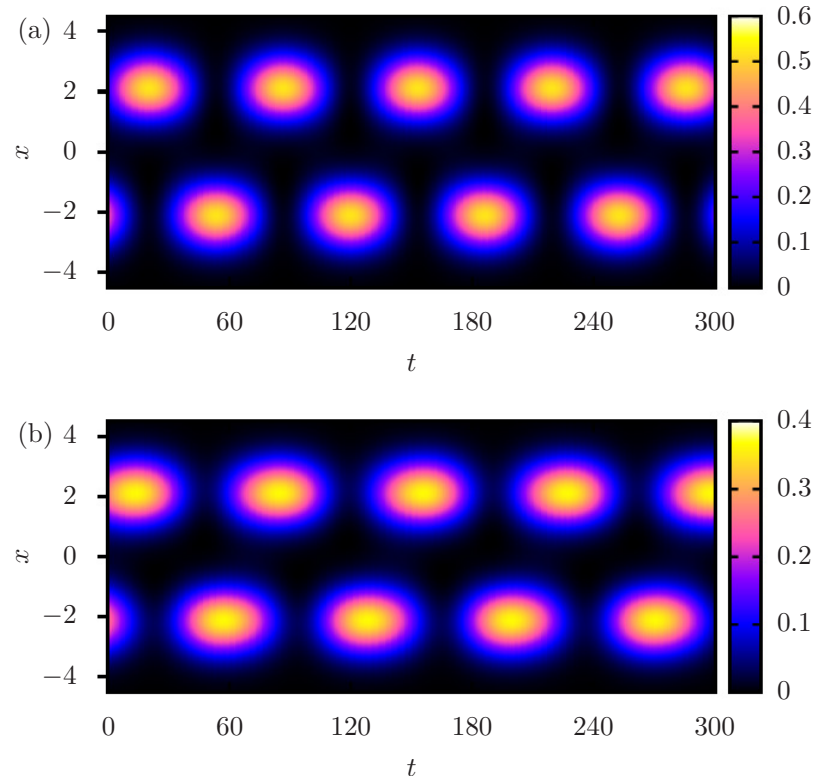

Figure 6. Evolution of the wave packet (14) in the $\mathcal{P} \mathcal{T}$ symmetric double well for nonvanishing nonlinearity $g=0.2, \varphi=\pi / 2$, and the gain-loss contributions $\Gamma=0$ (a) and $\Gamma=0.02(\mathrm{~b})$.

\subsection{TEMPORAl EVOLUtion AND THE SIGNIFICANCE OF "STATIONARY" SOLUTIONS WITH COMPLEX EIGENVALUES}

We first want to investigate the temporal evolution of condensate wave functions close to the stationary real eigenvalue solutions for values of $\Gamma$ below the appearance of the $\mathcal{P} \mathcal{T}$ broken states. Two examples of numerically exact propagations using the split operator method are given in Figure 6 for the initial wave packet

$$
\psi(x, t=0)=\frac{1}{\sqrt{2}}\left(\phi_{G S}(x)+\mathrm{e}^{\mathrm{i} \varphi} \phi_{E S}(x)\right)
$$

with the ground state $\phi_{G S}(x)$ and the excited state $\phi_{E S}(x)$. In this case one expects for linear systems the behavior that an oscillation of the probability amplitude between the two wells sets in. The frequency decreases with increasing $\Gamma$ until the oscillation stops at $\Gamma=\Gamma_{\mathrm{EP}}$ [11. This behavior is reproduced in Figure 6 i.e. the qualitative pattern of the motion is conserved in the nonlinear Gross-Pitaevskii equation (1). A quantitative analysis shows that the nonlinearity leads to slightly higher oscillation frequencies.

However, with the emergence of the additional $\mathcal{P} \mathcal{T}$ broken states we observe a qualitative change between the linear and nonlinear systems. As was mentioned above, the ground state becomes unstable in this regime. The relevance of this unstable character can be seen in Figure 7 , where the initial wave packet (14) is evolved for $g=0.2, \Gamma=0.03$, and the phase $\varphi=\pi$. Already during the first oscillation a complete deformation of the typical pattern is observed. Then the oscillation stops and changes into an unrestricted growth of the probability amplitude in the right well,
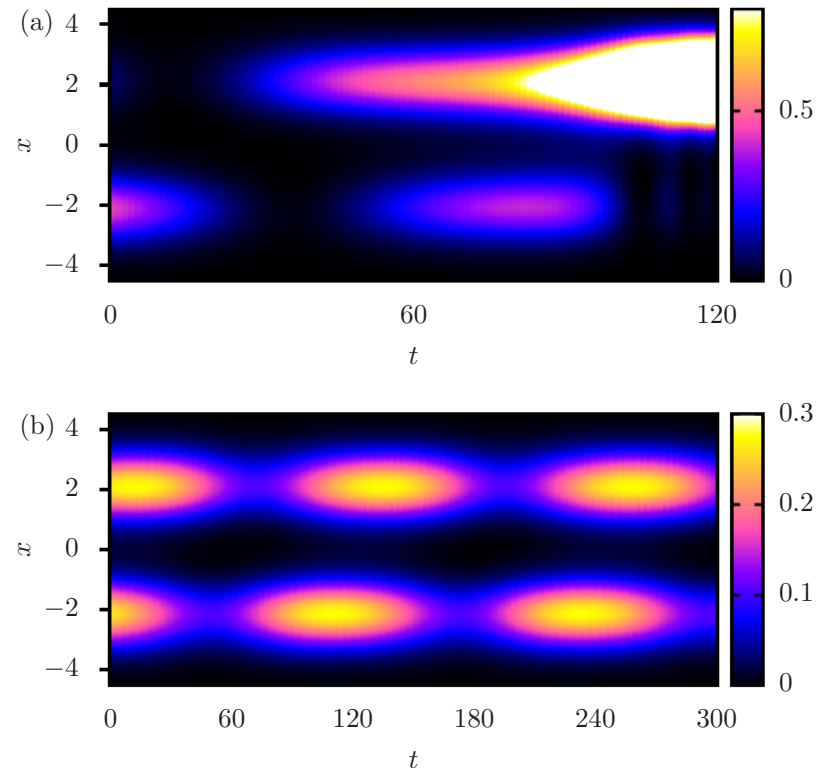

Figure 7. Evolution of the wave packet 14 in the $\mathcal{P T}$ symmetric double well for nonvanishing nonlinearity $g=0.2$ and the gain-loss contributions $\Gamma=0.03$ with phase $\varphi=\pi$ (a) and $\Gamma=0.04$ with $\varphi=\pi / 2$ (b).

the wave function "explodes". This behavior is not surprising. One of the superimposed states in (14 is unstable and the two $\mathcal{P} \mathcal{T}$ broken solutions with complex eigenvalues exist. One can expect that, in the nonlinear system, there is a considerable overlap of the time evolved wave function (14) with the $\mathcal{P} \mathcal{T}$ broken eigenstates, cf. also Figures 4 and 5 . Then, the eigenstate with positive imaginary part of the energy will always dominate for long times since it increases, and determines the further evolution. In a realistic situation an infinite growth of the probability amplitude will not appear. It has its origin in the $\mathcal{P} \mathcal{T}$ symmetric potential (4) and requires an infinite reservoir of atoms. At some point in time this description will break down.

The instability does not necessarily lead immediately to a destruction of the original oscillating behavior, as is shown for the example with $g=0.2$ and $\Gamma=0.04$ in Figure 7 p, i.e. very close to $\Gamma_{\mathrm{EP}}$. Even for a gain-loss influence larger than that chosen for Figure $7 \mathrm{a}$ it was possible to find a stable oscillation for the phase $\varphi=\pi / 2$. The behavior known from linear systems re-emerges. The probability amplitude almost pulsates in both wells with a low frequency.

From the dynamical point of view the solutions of the time-independent Gross-Pitaevskii equation (6) with complex eigenvalues have to be considered with some care. Strictly speaking, they lose their physical relevance. Due to the decay or growth of the probability amplitude mentioned above the nonlinear term $-g|\psi|^{2}$ in the Hamiltonian becomes explicitly time dependent. Thus, the states are not true stationary solutions of the time-dependent Gross-Pitaevskii equation (1). They are, however, still useful to indicate the 


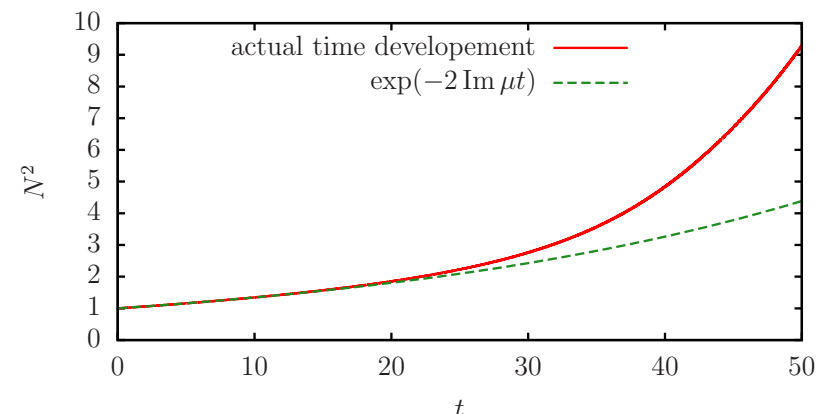

Figure 8. Temporal evolution of the norm of an initial complex eigenvalue state with $\operatorname{Im} \mu>0$. The parameters are $g=0.2$ and $\Gamma=0.03$.

temporal evolution of the condensate. For example, it was possible above to explain the explosion of the condensate wave function in Figure $7 \mathrm{a}$ by the presence of the growing $\mathcal{P} \mathcal{T}$ broken state.

Furthermore, it is possible to show that the complex eigenvalue solutions can still indicate the actual temporal evolution of an initial wave packet. In particular, for small times the prediction from the "stationary" complex eigenvalue solutions for the norm $N^{2}=\int|\psi|^{2} \mathrm{~d} x$ describes very well the true onset of the growth, as can be seen for an initial state with $\operatorname{Im} \mu>0$ in Figure 8

To investigate the correspondence of the complex eigenvalue solutions with the exact time integration for longer times we introduce the difference

$$
\begin{aligned}
D=\frac{1}{N}\left(\sqrt{\int_{\text {right well }}|\psi|^{2} \mathrm{~d} x}\right. & \\
- & \left.\sqrt{\int_{\text {left well }}|\psi|^{2} \mathrm{~d} x}\right),
\end{aligned}
$$

of the wave function's norm in the right and left wells. It tells us how the probability density is distributed in both wells. A positive value signalizes a higher probability density in the right well with gain, whereas a negative value indicates a concentration of the condensate's probability density in the left well with loss.

In Figure 9 we compare the norm difference $D$ of the correct temporal evolution with that of the complex eigenvalue solution with positive imaginary part, i.e. the growing state for the same parameters. Since the norm of the time evolved wave changes due to the gain-loss part of the potential we have to adapt the effective $g$ in the stationary Gross-Pitaevskii equation (6),

$$
g \rightarrow g N^{2}
$$

such that the two norm differences are comparable. In Figure 9a we plot $D$ for the situation depicted in Figure 7 With increasing time the state first decays and the norm drops. At low values of the norm the effective $g$ (16) assumes values at which only the true stationary states with real eigenvalues and $D=0$ exist. The latter property can be seen in the figure. However, at $t \approx 12$ the overlap with the growing
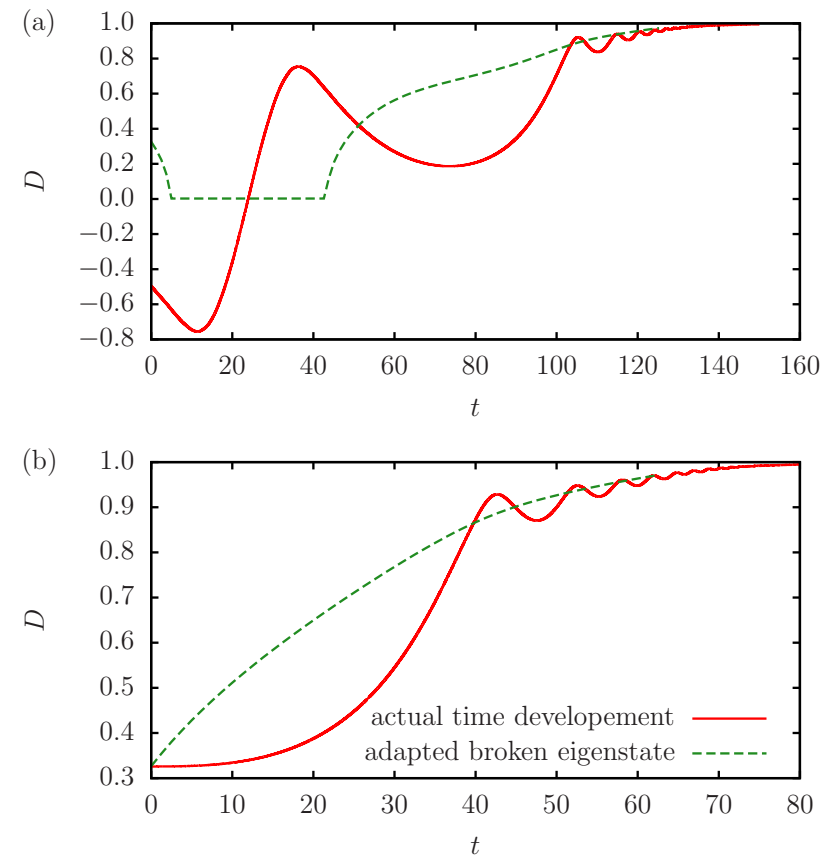

Figure 9. Comparison of the norm difference $D$ of the correct temporal evolution with that of complex eigenvalue solutions. In (a) the same situation as in Figure 7 is depicted, whereas (b) shows the evolution of the complex eigenvalue state with positive imaginary part. In both cases the norm difference follows the growing complex eigenvalue state for long times. $\Gamma$ was chosen to be 0.03 and the initial $g$ was 0.2 .

eigenstate wins and the norm starts to grow. After some oscillations of $D$, one observes for times $t>120$ that the norm differences of the two calculations agree more and more. This indicates that the wave function initially prepared in a superposition of two eigenstates evolves into the shape of the growing eigenstate for long times. That is, the complex eigenvalue solution with positive imaginary part still has a meaning for long times although it is not a true stationary solution of the time-dependent Gross-Pitaevskii equation (1). A similar behavior can be observed in Figure $9 \mathrm{~b}$, where the initial state was the growing complex eigenvalue solution of (6). Since we start with the increasing norm solution the agreement between both calculations sets in earlier.

\section{Conclusion}

The work presented in this article shows that $\mathcal{P} \mathcal{T}$ symmetric eigenfunctions exist in nonlinear quantum systems, in particular, in Bose-Einstein condensates described by the Gross-Pitaevskii equation. The solutions render the Hamiltonian itself $\mathcal{P} \mathcal{T}$ symmetric. A comparison of numerically exact calculations and a Gaussian approximation demonstrated that the Gaussian ansatz provides quantitatively well converged numerical results. A fully three-dimensional calculation of the condensate is possible, but is not necessary to describe the effects appearing due to the non-Hermitian 
gain-loss potential. It is even possible to extract quantitative values from a one-dimensional description of the condensate containing only the relevant direction, in which the $\mathcal{P} \mathcal{T}$ symmetric potential acts.

Solutions of the time-independent Gross-Pitaevskii equation with complex energy eigenvalues are found as well, and belong to eigenstates with broken $\mathcal{P} \mathcal{T}$ symmetry, destroying the Hamiltonian's symmetry. They have no direct physical meaning, since they are not true stationary states of the time-dependent Gross-Pitaevskii equation due to their complex energy eigenvalues indicating a growth or decay of the probability amplitude. However, one can observe that they influence the ground state. For nonvanishing nonlinearity $g$ the complex eigenvalue solutions bifurcate from the ground state when a critical value of the gain-loss parameter $\Gamma$ is exceeded. At this point the ground state becomes unstable, whereas the excited real eigenvalue solution is not affected by the appearance of the new states and stays stable as long as it exists.

The time evolution of the condensate showed that the eigensolutions of the time-independent GrossPitaevskii equation with complex eigenvalues may help to estimate the true temporal behavior of a condensate wave function. For small times, the imaginary part of the energy correctly describes the onset of the wave function's growth or decay. For large times, all initial wave functions tend to the state with positive imaginary part of the energy, which is located predominantly in the well with gain.

Certainly, there is a number of questions which still have to be answered. A better understanding of the nonlinearity's influence on the solutions is important. Analytically solvable matrix models might help to get a better insight. Furthermore, the relation of the threeand one-dimensional results should be investigated for higher values of the nonlinearity $g$.

A full analytical continuation of the non-analytical Gross-Pitaevskii equation should help to understand the change in the number of solutions observed in the eigenvalue diagrams. In a proper extension, the critical values of $\Gamma$ at which solutions appear or vanish should turn out to be bifurcation points.

It would be desirable to understand how a coherent in- or outcoupling of atoms can be understood on a microscopic level. This will be important for a realistic description of experimental situations. It is also possible to extend the model such that it can be understood as an embedding in a chain of potential wells. Here the gain-loss contributions can result from an effective description of a transport effect when only two wells somewhere in the middle of the chain are taken into account. Furthermore, it would be interesting to see how a gain-loss potential interacts with long-range inter-atomic interactions such as the dipole-dipole interaction leading often to qualitatively new effects [30].

\section{REFERENCES}

[1] Gross, E. P. Structure of a Quantized Vortex in Boson Systems. Nuovo Cimento 20, 454 (1961).

[2] Pitaevskii, L. P. Vortex Lines in an Imperfect Bose Gas. Sov. Phys. JETP 13, 451 (1961).

[3] Bender, C. M. and Boettcher, S. Real spectra in non-Hermitian Hamiltonians having PT symmetry. Phys. Rev. Lett. 80, 5243 (1998).

[4] Guo, A., et al. Observation of $\mathcal{P} \mathcal{T}$-Symmetry Breaking in Complex Optical Potentials. Phys. Rev. Lett. 103, 093902 (2009).

[5] Rüter, C. E., et al. Observation of parity-time symmetry in optics. Nat. Phys. 6, 192 (2010).

[6] Makris, K. G., et al. Beam Dynamics in $\mathcal{P} \mathcal{T}$ Symmetric Optical Lattices. Phys. Rev. Lett. 100, 103904 (2008).

[7] Makris, K. G., et al. $\mathcal{P} \mathcal{T}$-symmetric optical lattices. Phys. Rev. A 81, 063807 (2010).

[8] Ruschhaupt, A., et al. Physical realization of PT -symmetric potential scattering in a planar slab waveguide. J. Phys. A 38, L171 (2005).

[9] El-Ganainy, R., et al. Theory of coupled optical PT-symmetric structures. Opt. Lett. 32, 2632 (2007).

[10] Musslimani, Z., et al. Optical solitons in PT periodic potentials. Phys. Rev. Lett. 100, 30402 (2008).

[11] Klaiman, S., et al. Visualization of Branch Points in PT-Symmetric Waveguides. Phys. Rev. Lett. 101, 080402 (2008).

[12] Driben, R. and Malomed, B. A. Stability of solitons in parity-time-symmetric couplers. Opt. Lett. 36, 4323 (2011).

[13] Graefe, E. M., et al. A non-Hermitian $\mathcal{P} \mathcal{T}$ symmetric Bose-Hubbard model: eigenvalue rings from unfolding higher-order exceptional points. J. Phys. A 41, 255206 (2008).

[14] Graefe, E. M., et al. Mean-field dynamics of a non-Hermitian Bose-Hubbard dimer. Phys. Rev. Lett. 101, 150408 (2008).

[15] Graefe, E.-M., et al. Quantum-classical correspondence for a non-Hermitian Bose-Hubbard dimer. Phys. Rev. A 82, 013629 (2010).

[16] Musslimani, Z. H., et al. Analytical solutions to a class of nonlinear Schrödinger equations with $\mathcal{P} \mathcal{T}$-like potentials. J. Phys. A 41, 244019 (2008).

[17] Ramezani, H., et al. Unidirectional nonlinear $\mathcal{P} \mathcal{T}$-symmetric optical structures. Phys. Rev. A 82, 043803 (2010).

[18] Cartarius, H. and Wunner, G. Model of a $\mathcal{P} \mathcal{T}$-symmetric Bose-Einstein condensate in a $\delta$-function double-well potential. Phys. Rev. A 86, 013612 (2012).

[19] Jakubský, V. and Znojil, M. An explicitly solvable model of the spontaneous PT-symmetry breaking. Czech. J. Phys. 55, 1113 (2005).

[20] Mostafazadeh, A. Delta-function potential with a complex coupling. J. Phys. A 39, 13495 (2006). 
[21] Mostafazadeh, A. and Mehri-Dehnavi, H. Spectral singularities, biorthonormal systems and a

two-parameter family of complex point interactions. $J$. Phys. A 42, 125303 (2009).

[22] Mehri-Dehnavi, H., et al. Application of pseudo-Hermitian quantum mechanics to a complex scattering potential with point interactions. J. Phys. A 43, 145301 (2010).

[23] Jones, H. F. Interface between Hermitian and non-Hermitian Hamiltonians in a model calculation. Phys. Rev. D 78, 065032 (2008).

[24] Rau, S., et al. Pitchfork bifurcations in blood-cell-shaped dipolar Bose-Einstein condensates. Phys. Rev. A 81, 031605(R) (2010).

[25] Rau, S., et al. Variational methods with coupled Gaussian functions for Bose-Einstein condensates with long-range interactions. I. General concept. Phys. Rev. A 82, 023610 (2010).

[26] Rau, S., et al. Variational methods with coupled Gaussian functions for Bose-Einstein condensates with long-range interactions. II. Applications. Phys. Rev. A 82, 023611 (2010).

[27] McLachlan, A. D. A variational solution of the timedependent Schrödinger equation. Mol. Phys. 8, 39 (1964).

[28] Dast, D., et al. A Bose-Einstein condensate in a $\mathcal{P} \mathcal{T}$ symmetric double well. Fortschr. Phys. 61, 124 (2013).

[29] Press, W. H., et al. Numerical Recipes in Fortran 77. Cambridge University Press, New York, second edition (1992).

[30] Lahaye, T., et al. The physics of dipolar bosonic quantum gases. Rep. Prog. Phys. 72, 126401 (2009). 